\title{
Robust secrecy beamforming for wireless information and power transfer in multiuser MISO communication system
}

Wei Wu ${ }^{1 *}$ and Baoyun Wang ${ }^{1,2}$

\begin{abstract}
In this paper, we study the max-min fairness for robust secrecy beamforming design in a multiuser MISO communication system with simultaneous wireless information and power transfer (SWIPT). In particular, the imperfect channel state information (CSI) and power splitting information receiver (IR) are taken into account. We model the design as an optimization problem which maximizes the minimum harvested energy among the multi-antenna energy receivers (ERs). Besides, both the secure communication requirement and the lowest electrical energy storage threshold at IR must be guaranteed in our formulated optimization problem. The considered max-min problem is non-convex and hard to tackle. By introducing the technique of semidefinite relaxation (SDR), we prove the tightness of this relaxation and obtain the global optimal solution of our original optimization problem. Moreover, a suboptimal beamforming design scheme is proposed with low computational complexity. Simulation results show that our proposed robust schemes are more efficient than other schemes in terms of energy harvesting and transmit power saving.
\end{abstract}

Keywords: Robust secrecy beamforming; Wireless information and power transfer; Multi-input-single-output (MISO); Physical layer security

\section{Introduction}

The rapid growth of wireless communication requirement brings abundant energy consumption, which leads to the conventional battery-powered wireless communication devices encountering a bottleneck in providing sufficient power. Traditionally, we can harvest plentiful renewable energy from the solar, wind and tide, etc. However, the technique which we employed in collecting these energy may not be suitable for our portable communication devices. On the other hand, simultaneous wireless information and power transfer (SWIPT), which is regarded as a potential feasible technique to overcome this bottleneck, has attracted great interest of the researchers in recent years [1-5]. Using this technology, the battery-powered wireless communication devices can harvest energy from the ambient radio frequency (RF) signals to prolong their

\footnotetext{
*Correspondence: bywunjupt@foxmail.com

${ }^{1}$ College of Communication and Information, Nanjing University of Posts and Telecommunications, Xinmofan Road 66, 210003 Nanjing, China Full list of author information is available at the end of the article
}

lifetime. In particular, for a three-node multiple antenna SWIPT system, the optimal precoder was designed to achieve information and energy transmission trade-off [1]. The similar secrecy rate and power transfer trade-off problem between the IR and ERs was investigated in [3].

It is worth noting that all above works which considered the systems with SWIPT are under the assumption of perfect channel state information (CSI). However, in practice, it is hard to obtain perfect CSI at the transmitter due to the signal transmission delay, quantization errors, and channel breakdowns. Furthermore, as we all know, the perfect CSI methods are quite sensitive to the channel uncertainties. Therefore, some systems are constructed under imperfect channel realization (e.g., [6-8]). In particular, [6] and [7] studied the secure transmission with wiretap channel for SWIPT in the MISO broadcast system. Moreover, two kinds of suboptimal solutions are proposed to achieve the lower bound of robust secrecy rate since the optimal solution is hard to acquire in [7]. Both the system with perfect and imperfect CSIs are studied in [8], where the optimal power allocation scheme

\section{是 Springer}


under the dual use of ERs are derived. In [9], the authors investigated the optimal resource allocation design for the multiuser MISO system with SWIPT, the artificial noise [10] is incorporated to facilitate the energy harvesting at ER. As far as the authors know, most of the previous works on power transfer either focus on the optimization of transmit power of transmitter or secrecy capacity of IR. And they always assume that the receivers are equipped with single antenna or the CSIs are perfectly known at transmitter. These motivate us to research the rarely considered problem of fair power harvesting among all ERs which are equipped with multi-antenna. Besides, the CSIs of transmitter-to-ER links are imperfect.

In this paper, we concentrate on the optimal secrecy beamforming design for SWIPT in a multiuser MISO communication system with multiple multi-antenna ERs. The CSIs between transmitter and ERs are assumed to be imperfect. Besides, the power splitting receivers [11] and the AN-aided transmit strategy are taken into account. We aim to, under the constraints of secure communication requirement and the lowest electrical energy storage threshold at IR, maximize the minimum harvested energy among the multi-antenna ERs by jointly optimizing the transmit beamforming vectors, AN covariance, and power splitting ratio. The design of interest results in a nonconvex problem, which can be solved through the 'separable SDR' [12], and the resulting solution is proved to be the global optimal solution. Besides, we propose a suboptimal robust beamforming design scheme with smaller available solution set for power transfer and information decoding.

The contributions of this paper are summarized as follows:

- We studied the problem of fair energy harvesting among all ERs by considering imperfect CSI and power splitting IR in the multiuser MISO communication system. An optimal secrecy beamforming design scheme was proposed to achieve this fair energy transfer to ERs.

- We proved the tightness of the adopted SDR technique in our optimal design scheme by exploiting the structure of resulting solution and obtained the global optimal solution of our original optimization problem.

- We proposed a suboptimal secrecy beamforming design scheme by simplifying the analysis of the solution of original problem. Also, we proved the tightness of the adopted SDR programming in our suboptimal scheme.

Notations: In this paper, the bold capital and lowercase letters are used to denote matrices and vectors, respectively. $\varepsilon\{\cdot\},(\cdot)^{H}, \operatorname{Tr}(\cdot)$, rank $(\cdot)$, indicate the expectation, Hermitian transpose, trace, and rank, respectively.
$\mathbf{I}_{N}$ denotes the $N \times N$ identity matrix; $\mathbb{C}^{N \times M}$ and $\mathbb{H}_{+}^{N}$ denote the $N \times M$ complex matrices and $N \times N$ Hermitian matrices, respectively. $\|\cdot\|_{2}$ means the Euclidean norm of a vector while $\|\cdot\|_{F}$ means the Frobenius norm of a matrix; null (.) denotes the null space of a vector or matrix. $\mathbf{x} \sim \mathcal{C N}(\mu, \Lambda)$ means the vector $\mathbf{x}$ is a complex Gaussian variable with mean $\mu$ and covariance $\Lambda$.

\section{System model and problem formulation}

\subsection{System model}

Let us consider a downlink multiuser MISO communication system which consists of a transmitter and two kinds of receivers, namely one information receiver (IR) and $K>1$ energy receivers (ERs), cf. Fig. 1 . The transmitter is equipped with $N>1$ antennas while the information receiver has only one antenna and each energy receivers has $M>1$ antennas. We denote the channel vector between the transmitter and IR as $\mathbf{h} \in \mathbb{C}^{N \times 1}$ and the channel matrix between transmitter and the $k$ th $\mathrm{ER}$ as $\mathbf{G}_{k} \in \mathbb{C}^{N \times M}$. In this paper, we take into account the channel uncertainties for the transmitter-to-ER links while the CSI of the transmitter-to-IR link is perfectly known at transmitter. The CSI of the link between transmitter and the $k$ th ER is modeled as [13] $\mathbf{G}_{k}=\overline{\mathbf{G}}_{k}+\Delta \mathbf{G}_{k}$, where $\overline{\mathbf{G}}_{k}$ is the channel estimate values of the transmitter while $\Delta \mathbf{G}_{k}$ denotes the channel estimate error. The error is assumed to be bounded in a deterministic region: $\left\|\Delta \mathbf{G}_{k}\right\|_{F}=\|$ $\mathbf{G}_{k}-\overline{\mathbf{G}}_{k} \|_{F} \leq \varepsilon_{e, k}, \forall k$, for some $\varepsilon_{e, 1}, \ldots, \varepsilon_{e, K}>0$.

The transmitter transmits the information signals to the IR and energy signals to the ER at the same time. To ensure a secure communication at IR and facilitate the energy harvesting at the ER, artificial noise signals are also generated by our transmitter. As a result, we present the transmit signal vector as

$$
\mathbf{x}=\mathbf{w} s^{I}+\sum_{k=1}^{K} \mathbf{v}_{k} s_{k}^{E}+\mathbf{v}_{0},
$$

where $s^{I}$ and $s_{k}^{E}$ are the signals intended for IR and ERs, respectively. As usual, we assume that $\varepsilon\left\{\left|s^{I}\right|^{2}\right\}=1$ and $\varepsilon\left\{\left|s_{k}^{E}\right|^{2}\right\}=1, \forall k . \mathbf{w} \in \mathbb{C}^{N \times 1}$ and $\mathbf{v}_{k} \in \mathbb{C}^{N \times 1}$ are the information beam and energy beam, respectively. $\mathbf{v}_{0} \in \mathbb{C}^{N \times 1}$ is the artificial noise (AN) vector generated by the transmitter; we model it as $\mathbf{v}_{0} \sim \mathcal{C N}\left(\mathbf{0}, \mathbf{V}_{0}\right)$, where $\mathbf{V}_{0} \in$ $\mathbb{H}_{+}^{N}, \mathbf{V}_{0} \succeq \mathbf{0}$. Given the transmit signal $\mathbf{x}$, we model the received signals at IR and the $k$ th ER as

$y_{I}=\mathbf{h}^{H} \mathbf{x}+n_{I}$ and $\mathbf{y}_{E, k}=\mathbf{G}_{k}^{H} \mathbf{x}+\mathbf{n}_{E, k}, \forall k \in\{1, \ldots, K\}$, respectively. Where $n_{I} \sim \mathcal{C N}\left(0, \sigma_{I}^{2}\right)$ and $\mathbf{n}_{E, k} \sim$ $\mathcal{C N}\left(\mathbf{0}, \sigma_{E}^{2} \mathbf{I}_{M}\right)$ are the additive White Gaussian noises (AWGN) caused by the receive antennas of IR and ER, respectively. $\sigma_{I}^{2}$ and $\sigma_{E}^{2}$ denote the noise power of each other. 


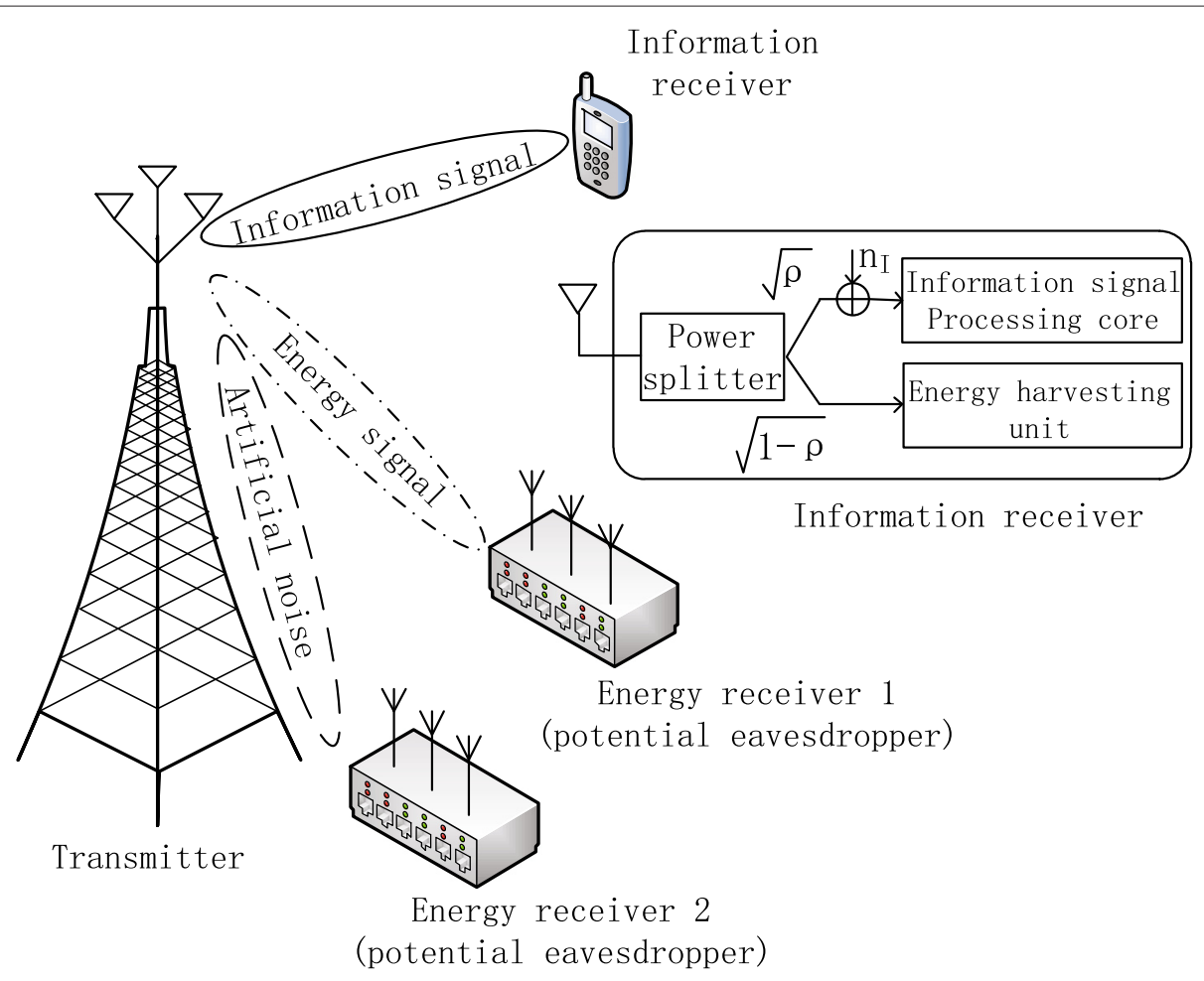

Fig. 1 Multiuser downlink communication system model with one single antenna information receiver and $K=2$ multi-antenna energy receivers

\subsection{Problem formulation}

In this considered system, the ERs can harvest energy from all the baseband signals transmitted from the transmitter. So, we model the harvested energy at the $k$ th ER as

$$
\begin{aligned}
E_{\mathrm{ER}, k} & =\eta_{k}\left(\left\|\mathbf{G}_{k}^{H} \mathbf{w}\right\|^{2}+\sum_{i=1}^{K}\left\|\mathbf{G}_{k}^{H} \mathbf{v}_{i}\right\|^{2}+\left\|\mathbf{G}_{k}^{H} \mathbf{v}_{0}\right\|^{2}\right) \\
& =\eta_{k} \operatorname{Tr}\left[\mathbf{G}_{k}^{H}\left(\mathbf{W}+\mathbf{V}+\mathbf{V}_{0}\right) \mathbf{G}_{k}\right], \forall k
\end{aligned}
$$

where $0<\eta_{k}<1$, a constant, denotes the energy harvesting efficiency when the ER intends to convert the received radio signals into electrical energy; $\mathbf{W}=\mathbf{w} \mathbf{w}^{H}$ and $\mathbf{V}=$ $\sum_{i=1}^{K} \mathbf{v}_{i} \mathbf{v}_{i}^{H}$

Remark 1. The dual use of artificial noise is advocated to facilitate the energy transfer and provide security.

As depicted in Fig. 1, the IR receives the information signals transmitted from the transmitter, and the received SINR can be written as

$$
\Gamma_{I}=\frac{\rho\left|\mathbf{h}^{H} \mathbf{w}\right|^{2}}{\rho\left|\mathbf{h}^{H} \mathbf{v}_{0}\right|^{2}+\rho \sum_{i=1}^{K}\left|\mathbf{h}^{H} \mathbf{v}_{i}\right|^{2}+\sigma_{I}^{2}}=\frac{\rho \operatorname{Tr}(\mathbf{H W})}{\rho \operatorname{Tr}\left(\mathbf{H} \mathbf{V}_{0}\right)+\rho \operatorname{Tr}(\mathbf{H V})+\sigma_{I}^{2}},
$$

where $0 \leq \rho \leq 1$ denotes the power splitting ratio since the power has been split into two parts, one part is used for decoding information signals and another part is preserved for future use; $\mathbf{H}=\mathbf{h} \mathbf{h}^{H}$. After this, the stored energy of IR can be represented as

$$
\begin{aligned}
E_{I} & =(1-\rho) \eta\left|\mathbf{h}^{H} \mathbf{w}\right|^{2}+(1-\rho) \eta\left(\left|\mathbf{h}^{H} \mathbf{v}_{0}\right|^{2}+\sum_{i=1}^{K}\left|\mathbf{h}^{H} \mathbf{v}_{i}\right|^{2}\right) \\
& =(1-\rho) \eta \operatorname{Tr}(\mathbf{H W})+(1-\rho) \eta\left[\operatorname{Tr}\left(\mathbf{H} \mathbf{V}_{0}\right)+\operatorname{Tr}(\mathbf{H V})\right],
\end{aligned}
$$

where $\eta$ is the same as $\eta_{k}$.

Without loss of generality, the ERs can also serve as an eavesdropper to decode the information for the IR. The SINR at the kth ER is given by

$$
\Gamma_{\mathrm{ER}, k}=\frac{\left\|\mathbf{G}_{k}^{H} \mathbf{w}\right\|^{2}}{\left\|\mathbf{G}_{k}^{H} \mathbf{v}_{0}\right\|^{2}+\sigma_{E}^{2}}=\frac{\operatorname{Tr}\left(\mathbf{G}_{k}^{H} \mathbf{W} \mathbf{G}_{k}\right)}{\operatorname{Tr}\left(\mathbf{G}_{k}^{H} \mathbf{V}_{0} \mathbf{G}_{k}\right)+\sigma_{E}^{2}}, \forall k .
$$

Remark 2. For the case that the energy beams carry no information but only serve as the pseudorandom signals which can be cancelled with a cancellation operation at each ER [14], thus the energy beams cause no interference at ER besides the AN.

The purpose of this system is to maximize the minimum harvest energy among all the $K$ ERs such that the QoS is provided for secure communication and the energy stored in the device of IR is above a given threshold while the total transmit power is under a given threshold. Hence, 
consider the worst-case CSIs, the optimization problem of secrecy beamforming design can be written as

$$
\begin{array}{cl}
\max _{\mathbf{W}, \mathbf{V}, \mathbf{V}_{0},}, k, \Delta \mathbf{G}_{k} & \eta_{k} \operatorname{Tr}\left[\mathbf{G}_{k}^{H}\left(\mathbf{W}+\mathbf{V}+\mathbf{V}_{0}\right) \mathbf{G}_{k}\right] \\
\text { s.t. } & C 1: \Gamma_{I} \geq r, \\
& C 2: \max _{\Delta \mathbf{G}_{k}} \Gamma_{\mathrm{ER}, k} \leq r_{k}, \forall k \\
& C 3: \mathrm{E}_{I} \geq P_{\min }, \\
& C 4: \operatorname{Tr}\left(\mathbf{W}+\mathbf{V}+\mathbf{V}_{0}\right) \leq P
\end{array}
$$

where $r$ is the minimum required SINR at IR, $r_{k}$ is the maximum required SINR at the $k$ th ER, $P_{\min }$ is the lower limit of the energy stored in IR, and $P$ is the upper bound of the total transmit power at the transmitter. By introducing an auxiliary optimization variable $t$ and rewriting the fractional constraints, problem (1) can be reformulated as a SDP problem as follow:

$$
\begin{gathered}
\max _{\mathbf{W}, \mathbf{V}, \mathbf{V}_{0}, \rho, t} t \\
\text { s.t. } C 1: \operatorname{Tr}\left[\left(\mathbf{W}-r \mathbf{V}-r \mathbf{V}_{0}\right) \mathbf{H}\right] \geq \frac{r \sigma_{I}^{2}}{\rho}, \\
C 2: \max _{\Delta \mathbf{G}_{k}} \operatorname{Tr}\left[\mathbf{G}_{k}^{H}\left(\mathbf{W}-r_{k} \mathbf{V}_{0}\right) \mathbf{G}_{k}\right] \leq r_{k} \sigma_{k}^{2}, \forall k, \\
C 3: \operatorname{Tr}\left[\left(\mathbf{W}+\mathbf{V}+\mathbf{V}_{0}\right) \mathbf{H}\right] \geq \frac{P_{\min }}{(1-\rho) \eta}, \\
C 4: \operatorname{Tr}\left(\mathbf{W}+\mathbf{V}+\mathbf{V}_{0}\right) \leq P, \\
C 5: \min _{\Delta \mathbf{G}_{k}} \eta_{k} \operatorname{Tr}\left[\mathbf{G}_{k}^{H}\left(\mathbf{W}+\mathbf{V}+\mathbf{V}_{0}\right) \mathbf{G}_{k}\right] \geq t, \forall k, \\
C 6: 0 \leq \rho \leq 1, \quad C 7: \mathbf{W}, \mathbf{V}, \mathbf{V}_{0} \geq 0, \\
C 8: \operatorname{rank}(\mathbf{W})=1,
\end{gathered}
$$

It is obvious that problem (2) is not a convex SDP problem because of constraint $\mathrm{C} 8$. Then, by adopting SDP relaxation (SDR) technique [15], remove $C 8$, we obtain a convex SDR problem. This convex SDR problem of (2) is given by

$$
\begin{array}{ll}
\max _{\mathbf{W}, \mathbf{V}, \mathbf{V}_{0}, \rho, t} & t \\
\text { s.t. } & C 1, C 2, C 3, C 4, C 5, C 6, C 7 .
\end{array}
$$

\section{Solution of the optimization problem}

In this section, we aim to turn problem (3) to a tractable convex problem and to find a rank-one optimal solution W by studying the solution structure of (3).

Problem (3) is intractable for robust downlink beamforming design because of the semi-infiniteness of constraints $\mathrm{C} 1, \mathrm{C} 2, \mathrm{C} 3$, and $\mathrm{C} 5$. To facilitate the solution, we consider to turn these constrains into linear matrix inequalities (LMIs) [16] by using the S-procedure method:

Lemma 1. (S - procedure [17]): Let a function $f_{m}(\mathbf{x}), m=\{1,2\}$, be defined as

$$
f_{m}(\mathbf{x})=\mathbf{x}^{H} \mathbf{A}_{m} \mathbf{x}+2 \operatorname{Re}\left\{\mathbf{b}_{m}^{H} \mathbf{x}\right\}+\mathbf{c}_{m},
$$

where $\mathbf{x} \in \mathbb{C}^{N \times 1}, \mathbf{A}_{m} \in \mathbb{H}_{+}^{N}, \mathbf{b}_{m} \in \mathbb{C}^{N \times 1}$ and $c_{m} \in \mathcal{R}$. then, the deduction $f_{1}(\mathbf{x}) \leq 0 \Rightarrow f_{2}(\mathbf{x}) \leq 0$ holds if and only if there exists $a \lambda \geq 0$ such that

$$
\lambda\left[\begin{array}{ll}
\mathbf{A}_{1} & \mathbf{b}_{1} \\
\mathbf{b}_{1}^{H} & c_{1}
\end{array}\right]-\left[\begin{array}{ll}
\mathbf{A}_{2} & \mathbf{b}_{2} \\
\mathbf{b}_{2}^{H} & c_{2}
\end{array}\right] \succeq \mathbf{0}
$$

provided that there exists a point $\hat{\mathbf{x}}$ such that $f_{1}(\hat{\mathbf{x}})<0$.

For the purpose of applying Lemma 1 to problem (3), we substitute $\mathbf{G}_{k}=\overline{\mathbf{G}}_{k}+\Delta \mathbf{G}_{k}$ into $C 2$ and rewrite $\mathrm{C} 2$ as the following implication:

$$
\begin{aligned}
\Delta \mathbf{g}_{k}^{H} \Delta \mathbf{g}_{k} \leq \varepsilon_{e, k}^{2} & \Rightarrow \Delta \mathbf{g}_{k}^{H} \Delta_{2} \Delta \mathbf{g}_{k}+2 \operatorname{Re}\left\{\overline{\mathbf{g}}_{k}^{H} \Delta_{2} \Delta \mathbf{g}_{k}\right\} \\
& +\overline{\mathbf{g}}_{k}^{H} \Delta_{2} \overline{\mathbf{g}}_{k}-r_{k} \varepsilon_{e, k}^{2} \leq 0,
\end{aligned}
$$

where $\Delta_{2}=\overline{\mathbf{W}}-r_{k} \overline{\mathbf{V}}_{0}, \overline{\mathbf{W}}=\mathbf{I}_{M} \otimes \mathbf{W}, \overline{\mathbf{V}}_{0}=\mathbf{I}_{M} \otimes \mathbf{V}_{0}$ and $\overline{\mathbf{g}}_{k}=\operatorname{vec}\left(\overline{\mathbf{G}}_{k}\right)$. Using Lemma 1, we re-express (4) as

$$
\begin{aligned}
& C 2: \mathbf{T}_{E R, k}\left(\mathbf{W}, \mathbf{V}_{0}, \tilde{\lambda}_{k}\right) \\
& =\left[\begin{array}{cc}
\tilde{\lambda}_{k} \mathbf{I}_{N M}-\Delta_{2} & -\Delta_{2} \overline{\mathbf{g}}_{k} \\
-\overline{\mathbf{g}}_{k}^{H} \Delta_{2} & -\overline{\mathbf{g}}_{k}^{H} \Delta_{2} \overline{\mathbf{g}}_{k}+r_{k} \sigma_{E}^{2}-\tilde{\lambda}_{k} \varepsilon_{e, k}^{2}
\end{array}\right] \succeq \mathbf{0},
\end{aligned}
$$

for some $\tilde{\lambda}_{k} \geq 0, k=1, \ldots, K$. Similarly, the C5 is equivalent to the following expression:

$$
\begin{aligned}
& C 5: \mathbf{M}_{E R, k}\left(\mathbf{W}, \mathbf{V}, \mathbf{V}_{0}, \lambda_{k}\right) \\
& =\left[\begin{array}{cc}
\lambda_{k} \mathbf{I}_{N M}+\Delta_{5} & \Delta_{5} \overline{\mathbf{g}}_{k} \\
\overline{\mathbf{g}}_{k}^{H} \Delta_{5} & \overline{\mathbf{g}}_{k}^{H} \Delta_{5} \overline{\mathbf{g}}_{k}-\frac{t}{\eta}-\lambda_{k} \varepsilon_{e, k}^{2}
\end{array}\right] \succeq \mathbf{0},
\end{aligned}
$$

for some $\lambda_{k} \geq 0, k=1, \ldots, K$ and $\Delta_{5}=\overline{\mathbf{W}}+\overline{\mathbf{V}}+\overline{\mathbf{V}}_{0}$, where $\overline{\mathbf{V}}=\mathbf{I}_{M} \otimes \mathbf{V}$. Substituting (5) and (6) back into (3), we obtain the following SDR problem:

$$
\begin{aligned}
\max & t \\
\mathbf{W}, \mathbf{V}, \mathbf{V}_{0}, \rho, t,\left\{\lambda_{k}\right\},\left\{\tilde{\lambda}_{k}\right\} & \\
\text { s.t. } & C 1, \mathrm{C} 3, \mathrm{C} 4, \mathrm{C} 6, \mathrm{C} 7, \\
& C 2: \mathbf{T}_{\mathrm{ER}, k}\left(\mathbf{W}, \mathbf{V}_{0}, \tilde{\lambda}_{k}\right) \geq \mathbf{0}, \forall k, \\
& C 5: \mathbf{M}_{\mathrm{ER}, k}\left(\mathbf{W}, \mathbf{V}, \mathbf{V}_{0}, \lambda_{k}\right) \geq \mathbf{0}, \forall k, \\
& C 9:\left\{\lambda_{k}\right\},\left\{\tilde{\lambda}_{k}\right\} \geq 0, \forall k .
\end{aligned}
$$

We note that the relaxed SDP problem (7) is a common convex optimization problem which can be solved efficiently by the existing solvers such as SDPT3 [18] and SeDuMi [19]. If the obtained solution $\mathbf{W}$ is rank one, then the SDR optimization problem (7) shares the same optimal solution and objective value with the original optimization problem (1). As a result, we work out the information beam $\mathbf{w}$ by performing eigenvalue decomposition on W. However, as we all know, the obtained solution $\mathbf{W}$ for the SDR problem (7) may not be rank one, so the resulting solution is not the optimal solution of the original optimization problem. In the following, we will introduce a theorem to reveal the tightness of the 
relaxed SDP problem (7). Then, by analyzing the structure of the resulting solution, we construct an optimal solution for problem (7) with rank $(\mathbf{W})=1$. Furthermore, we propose a suboptimal secrecy beamforming design scheme with lower computational complexity by simplifying the optimality condition of constructing the rank-one solution.

\subsection{Optimal solution}

In this subsection, we will reveal the tightness of our proposed relaxed SDP problem (7) by introducing a theorem which shares the similar idea as the Proposition 4.1 in [3].

Before the theorem, we first define a crucial formula which serves as a cornerstone in revealing the tightness of our proposed relaxed SDP problem. Define:

$$
\mathbf{B}_{1}^{*}=-\alpha^{*} \mathbf{I}_{N}+\sum_{k=1}^{K} \sum_{l=1}^{M}\left(\mathbf{z}_{e, k}^{*(l, l)}-\mathbf{U}_{e, k}^{*(l, l)}\right)
$$

and $r_{1}=\operatorname{rank}\left(\mathbf{B}_{1}^{*}\right)$. Where $\mathbf{Z}_{e, k}^{*(l, l)} \in \mathbb{H}_{+}^{N}$ and $\mathbf{U}_{e, k}^{*(l, l)} \in$ $\mathbb{H}_{+}^{N}$ are the $l$ th entry matrices on the diagonal of $\tilde{\mathbf{G}}_{k} \mathbf{X}_{k} \tilde{\mathbf{G}}_{k}^{H} \in \mathbb{H}_{+}^{N M}$ and $\tilde{\mathbf{G}}_{k} \mathbf{Y}_{\mathbf{k}} \tilde{\mathbf{G}}_{k}^{H} \in \mathbb{H}_{+}^{N M}$, respectively; where $\tilde{\mathbf{G}}_{k}=\left[\mathbf{I}_{N M} \overline{\mathbf{g}}_{k}\right]$, and $\mathbf{X}_{k}$ and $\mathbf{Y}_{k}$ are the Lagrange dual variables with respect to $\mathbf{M}_{\mathrm{ER}, k}\left(\mathbf{W}, \mathbf{V}, \mathbf{V}_{0}, \lambda_{k}\right)$ and $\mathbf{T}_{\mathrm{ER}, k}\left(\mathbf{W}, \mathbf{V}_{0}, \tilde{\lambda}_{k}\right)$, respectively. Furthermore, we model the orthogonal basis of the null space of $\mathbf{B}_{1}^{*}$ as $\mathbf{N}_{1} \in$ $\mathbb{C}^{N \times\left(N-r_{1}\right)}$, and $\pi_{1, n} \in \mathbb{C}^{N \times 1}, 1 \leq n \leq N-r_{1}$ as the $n$th column of $\mathbf{N}_{1}$. Upon that, we have the following theorem.

Theorem 1. The optimal solution $\left\{\mathbf{W}^{*}, \mathbf{V}^{*}, \mathbf{V}_{0}^{*}, \rho^{*}, t^{*}\right\}$ of problem (7) is characterized as follows:

1. The optimal solution $\mathbf{W}^{*}$ can be expressed as

$$
\mathbf{W}^{*}=\sum_{n=1}^{N-r_{1}} \mu_{n} \pi_{1, n} \pi_{1, n}^{H}+f \tau_{1} \tau_{1}^{H},
$$

where $\mu_{n} \geq 0, \forall n, f \geq 0$ and $\tau_{1} \in \mathbb{C}^{N \times 1},\left\|\tau_{1}\right\|_{2}=1$ satisfies $\tau_{1}^{H} \mathbf{N}_{1}=0$.

2. If the optimal solution $\mathbf{W}^{*}$ given in (9) has $\operatorname{rank}\left(\mathbf{W}^{*}\right)>1$, i.e., $\mu_{\mathrm{n}}>0, \exists n$. Then, we have another solution

$$
\begin{aligned}
& \widetilde{\mathbf{W}}^{*}=\mathbf{W}^{*}-\sum_{n=1}^{N-r_{1}} \mu_{n} \pi_{1, n} \pi_{1, n}^{H}=f \tau_{1} \tau_{1}^{H}, \\
& \tilde{\mathbf{V}}^{*}=\mathbf{V}^{*}+\sum_{n=1}^{N-r_{1}} \mu_{n} \pi_{1, n} \pi_{1, n}^{H}, \\
& \tilde{\mathbf{V}}_{0}^{*}=\mathbf{V}_{0}^{*}, \tilde{\rho}^{*}=\rho^{*}, \tilde{t}^{*}=t^{*},
\end{aligned}
$$

with $\widetilde{\mathbf{W}}^{*}$ serve as the new optimal solution and has $\operatorname{rank}\left(\widetilde{\mathbf{W}}^{*}\right)=1$.

Proof. : Please refer to Appendix 1.
With Theorem 1, the global optimal solution of problem (1) is achieved. First, we solve the SDR problem (7) via CVX and obtain the solution $\left\{\mathbf{W}^{*}, \mathbf{V}^{*}, \mathbf{V}_{0}^{*}, \rho^{*}, t^{*}\right\}$. If the information beamforming matrix satisfies $\operatorname{rank}\left(\mathbf{W}^{*}\right)=$ 1 , the solution turns out to be optimal. If not, i.e., $\operatorname{rank}\left(\mathbf{W}^{*}\right)>1$. Then, we format an alternative optimal solution $\left\{\widetilde{\mathbf{W}}^{*}, \widetilde{\mathbf{V}}^{*}, \widetilde{\mathbf{V}}_{0}^{*}, \tilde{\rho}^{*}, \tilde{t}^{*}\right\}$ in accordance with (10)-(12) with $\operatorname{rank}\left(\widetilde{\mathbf{W}}^{*}\right)=1$ and achieve the same objective value.

\subsection{Suboptimal downlink beamforming design}

As discussed in Appendix 1, it involves solving the complex dual optimization problem (18) to construct an optimal solution set $\left\{\mathbf{W}^{*}, \mathbf{V}^{*}, \mathbf{V}_{0}^{*}, \rho^{*}, t^{*}\right\}$ of the relaxed SDP problem (7) with $\operatorname{rank}\left(\mathbf{W}^{*}\right)=1$. Besides, the uncertainty of the value of $\operatorname{rank}\left(B_{1}^{*}\right)$ results in an obscure value of $\operatorname{rank}\left(\mathbf{W}^{*}\right)$. Therefore, an additional procedure is inevitable to construct an alternative optimal solution set $\left\{\widetilde{\mathbf{W}}^{*}, \widetilde{\mathbf{V}}^{*}, \widetilde{\mathbf{V}}_{0}^{*}, \tilde{\rho}^{*}, \tilde{t}^{*}\right\}$ with $\operatorname{rank}\left(\widetilde{\mathbf{W}}^{*}\right)=1$ when $\operatorname{rank}\left(\mathbf{W}^{*}\right)>1$. In this case, we propose a suboptimal secrecy downlink beamforming design scheme which achieves a rank-one optimal solution $\mathbf{W}$ with lower computational complexity.

Proposition 1. Consider $\mathbf{X}_{k}^{*}$ and $\mathbf{Y}_{k}^{*}$ as the optimal Lagrange dual multiplier matrixes of the relaxed SDP problem (7) associated with constraints $C 5$ and $C 2$, respectively. The condition $\sum_{k=1}^{K} \sum_{l=1}^{M}\left(\mathbf{U}_{e, k}^{*(l, l)}-\mathbf{Z}_{e, k}^{*(l, l)}\right) \succeq \mathbf{0}$ must be held to ensure $\operatorname{ran} k\left(\mathbf{W}^{*}\right)=1$.

Proof. Please refer to Appendix 2.

According to Proposition 1, we learn that the condition $\sum_{k=1}^{K} \sum_{l=1}^{M}\left(\mathbf{U}_{e, k}^{*(l, l)}-\mathbf{Z}_{e, k}^{*(l, l)}\right) \succeq \mathbf{0}$ holds when constraint C5 is inactive, i.e., $\mathbf{X}_{k}^{*}=\mathbf{0}$, or the optimization beamforming matrix $\mathbf{W}$ is independent of $\mathbf{C} 5$. Intuitively, the auxiliary optimization variable $t$ determines our optimal objective value, so constraint $\mathrm{C} 5$ must be active during our operation. With this knowledge, we assume $\mathbf{W}$ is independent of $\mathrm{C} 5$ and replace $\mathrm{C} 5$ with $\overline{C 5}$. Then, we form the following new optimization problem:

$$
\begin{aligned}
& \max _{\mathbf{W}, \mathbf{V}, \mathbf{V}_{0}, \rho, t} t \\
& \text { s.t. } \quad C 1, C 2, C 3, C 4, C 6, C 7, \\
& \overline{C 5}: \mathbf{M}_{\mathrm{ER}, k}\left(\mathbf{V}, \mathbf{V}_{0}, \lambda_{k}\right) \\
& =\left[\begin{array}{cc}
\lambda_{k} \mathbf{I}_{N M}+\overline{\mathbf{V}}+\overline{\mathbf{V}}_{0} & \left(\overline{\mathbf{V}}+\overline{\mathbf{V}}_{0}\right) \overline{\mathbf{g}}_{k} \\
\overline{\mathbf{g}}_{k}^{H}\left(\overline{\mathbf{V}}+\overline{\mathbf{V}}_{0}\right) & \overline{\mathbf{g}}_{k}^{H}\left(\overline{\mathbf{V}}+\overline{\mathbf{V}}_{0}\right) \overline{\mathbf{g}}_{k}-\frac{t}{\eta}-\lambda_{k} \varepsilon_{e, k}^{2}
\end{array}\right] \\
& =\left[\begin{array}{cc}
\lambda_{k} \mathbf{I}_{\mathrm{NM}} & \mathbf{0} \\
\mathbf{0} & -\frac{t}{\eta}-\lambda_{k} \varepsilon_{e, k}^{2}
\end{array}\right]+\widetilde{\mathbf{G}}_{k}^{H}\left(\overline{\mathbf{V}}+\overline{\mathbf{V}}_{0}\right) \widetilde{\mathbf{G}}_{k} \succeq 0, \forall k .
\end{aligned}
$$


Compared to constraint $\mathrm{C} 5$, it is obvious that $\overline{\mathrm{C} 5}$ neglects the contribution of information beam in terms of energy harvesting, i.e., $\mathbf{W}$ is wiped out. As a result, the new optimization problem (13) performs worse compared to problem (7). We note that the reformulated constraint $\overline{C 5}$ is convex, thus problem (13) can be solved efficiently through the aforementioned numerical solvers. Furthermore, it always has a rank-one optimal beamforming solution, i.e., $\operatorname{rank}(\mathbf{W})=\mathbf{1}$, which proves the tightness of the SDP relaxation.

\section{Simulation results}

In this section, we evaluate the performance of our proposed robust downlink beamforming design scheme for multiuser MISO communication system with imperfect CSI via simulation. We set the simulation parameters as $N=5, K=3, M=3, \mathrm{r}=10 \mathrm{~dB}, r_{k}=0 \mathrm{~dB}, P=$ $10 \mathrm{~mW}, P_{\min }=1 \mathrm{~mW}, \eta=0.5$ and $\sigma_{I}^{2}=\sigma_{E}^{2}=10^{-3}$. The channel entries associated with our system are randomly generated i.i.d. complex Gaussian variables which obey $\mathcal{C N}(0,1)$. All simulation results were achieved by an average of 1000 channel realizations.

\subsection{Average total harvested power}

To the imperfect CSI between the transmitter and ERs, we define $\alpha_{e, k}=\frac{\varepsilon_{e, k}}{\sqrt{E\left\{\left\|\bar{G}_{k}\right\|_{F}^{2}\right\}}}, k=1, \ldots, K$ as channel uncertainty ratio to evaluate the $k$ th channel estimate error. We will set $\alpha_{e, 1}=\ldots=\alpha_{e, K}=\alpha_{e}$ and choose $\alpha_{e}=0.05$, unless specified.

Figure 2 shows the average minimum harvested power at per ER versus ERs' channel uncertainty ratio $\alpha_{e}$. It is observed that both our proposed robust scheme and non-robust scheme meet the performance attenuation in terms of power harvesting with increased $\alpha_{e}$. The case

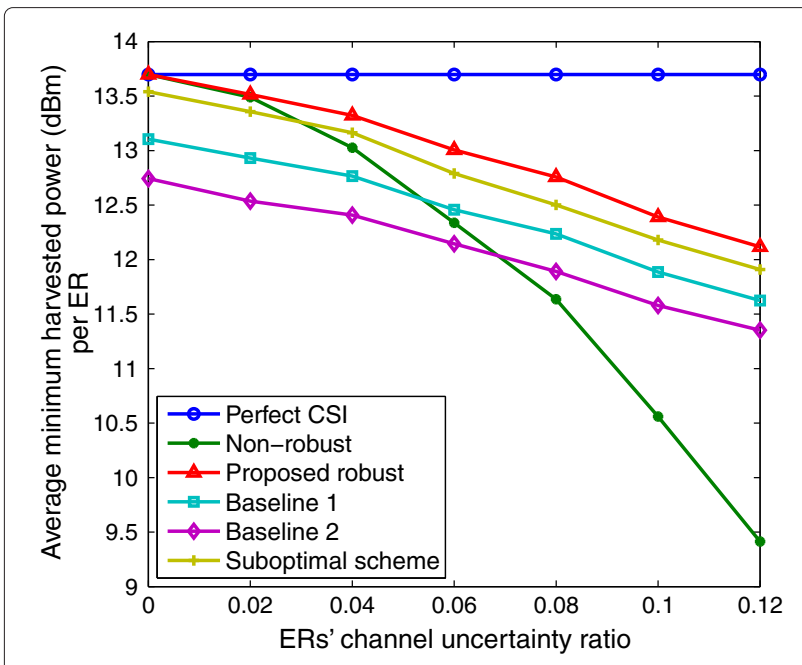

Fig. 2 Simulation 1. Average minimum harvested power per ER versus ERs' channel uncertainty ratio, $\alpha_{e}$ that the transmitter has perfect CSI, of course, is independent of $\alpha_{e}$. As depicted in the figure, our proposed robust downlink beamforming design performs better than the non-robust scheme, especially when $\alpha_{e}$ is large. This is attributed to the fact that the non-robust scheme is much sensitive to the channel uncertainty while our proposed robust scheme has a full utilization of the CSI of the transmitter-to-ER links. For a comprehensive comparison, Fig. 2 also depicts the performance of our proposed suboptimal scheme and two baseline schemes. For baseline scheme 1 , zero-forcing method is adopted to reduce the undesired interference to the IR. Particularly, we eliminate the interference, which from the energy beams and artificial noise, to IR by forcing the energy beamforming matrix $\mathbf{V}$ and noise covariance matrix $\mathbf{V}_{0}$ lie in the null space of channel $\mathbf{h}$ [20]. Moreover, we perform maximum ratio transmission (MRT) for conveying information to the IR. For baseline 2, we employ the same beamforming design scheme as baseline 1 besides that we fix the splitting ratio at $\rho=0.5$. From Fig. 2, we learn that our proposed suboptimal scheme still harvests lower power than our proposed optimal scheme owning to the fact that the former achieves the optimal objective value in a smaller feasible solution set. On the other hand, two baseline schemes achieve the optimal downlink beamforming design of each other with lower computational complexity at the expense of distinctly lower power harvesting. Furthermore, baseline scheme 1 acquire obvious performance gain compared with baseline scheme 2 thanks to the optimization of the splitting ratio $\rho$.

Figure 3 illustrates the average minimum harvested power per ER versus the total transmit power, $P$, at the transmitter for different beamforming design schemes and channel uncertainty ratios, $\alpha_{e}$. As expected, the

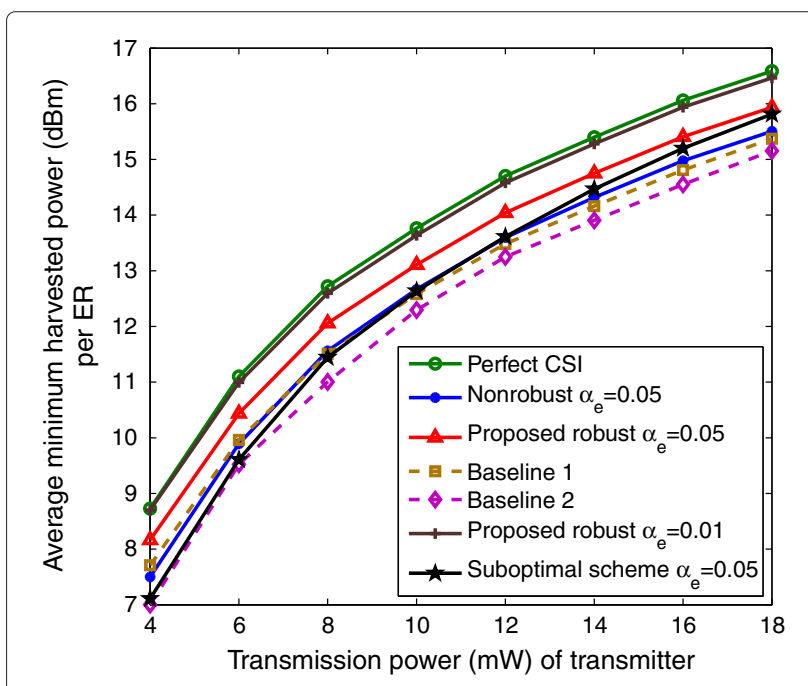

Fig. 3 Simulation 2. Average minimum harvested power per ER versus total transmit power, $P$ 
harvested power at ER increased with the total transmit power of the transmitter in different scenarios. From this figure, we learn intuitively that our proposed robust scheme harvests nearly the same power as the perfect CSI scenario with lower channel uncertainty ratio, cf. the curves 'Perfect CSI' and 'Proposed robust $\alpha_{e}=0.01$ '. On the other hand, it is observed that the performance gap between our proposed robust scheme and suboptimal scheme decreased with the increasing transmit power. This is owing to the fact that the increasing transmit power enlarges the feasible solution set of the suboptimal problem (13) which tend to be equal to the feasible solution set of problem (7). Besides, our proposed robust scheme still performs better than the non-robust scheme in terms of power harvesting with different transmit power.

Figure 4 illustrates the average minimum harvested power per ER versus the minimum required SINR of IR, $\Gamma_{I}$. As expected, the harvested power decreases with increasing $\Gamma_{I}$. This is attributed to the fact that it is a trade-off of our secrecy beamforming design between power harvesting of ER and the guarantee of secure communication of IR. In particular, the excessive power harvesting of ER comes at the expense of significantly low secrecy rate of IR which is proportional, with fixed $r_{k}$, to the SINR of IR, vice versa. Moreover, the harvested power degrades more significant in the high required SINR of IR regime due to the limited energy-rate region. Among all the considered schemes, with fixed $\alpha_{e}=0.05$, our proposed scheme still shows a better performance than other schemes. The gap between our proposed robust scheme and the suboptimal scheme enlarged with increasing SINR for the feasible solution set of the suboptimal scheme is inversely proportional to the required SINR of IR. The similar situation occurs between two baseline schemes

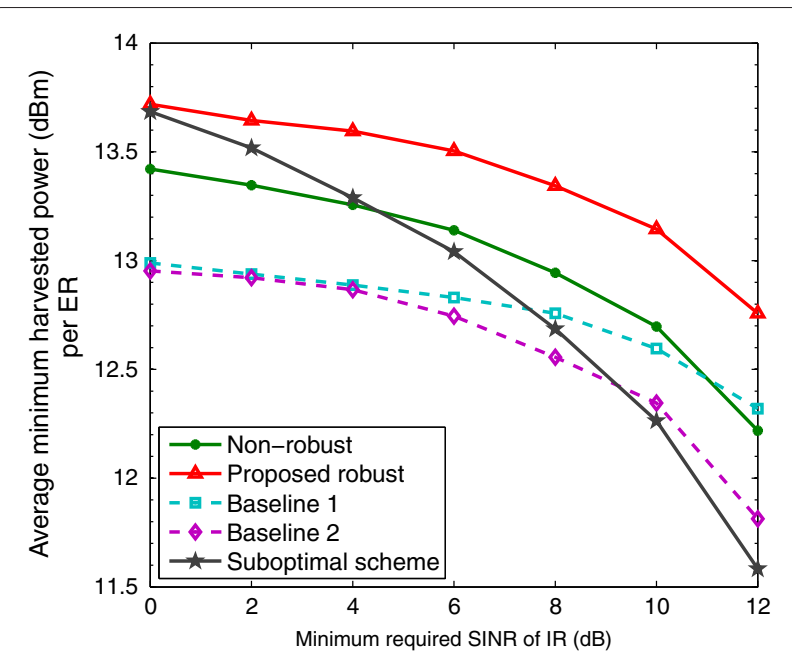

Fig. 4 Simulation 3. Average minimum harvested power per ER versus the minimum required SINR of $I R, \Gamma_{\text {, }}$ where the baseline scheme 2 performs even worse than baseline scheme 1 in high required SINR regime. This is own to the fact that the optimal power splitting ratio $\rho$ gradually deviated from 0.5 with increasing required SINR value.

Figure 5 illustrates the average harvested power per ER versus numbers of transmit antenna $\mathrm{N}$ with fixed transmit power $P$. From this figure, we observe that the minimum harvested power among the $K$ ERs increased with increasing number of transmit antennas. In fact, the degrees of freedom for beamforming design increases with the number of transmit antennas. Moreover, the higher the degrees of space freedom the more power efficiency for information and power beams. Thus, the minimum harvested power per ER is proportional to the number of transmit antennas. Focusing on the non-robust scheme, we see that it performs worse compared to our proposed robust scheme except the point $N=3$. This is caused by the fact that, with less transmit antennas, our proposed robust scheme is not able to fully use the CSI of all transmitter-to-ER channels. With this, we always assume that the transmitter has more antennas than each receiver in our system. For the two baseline schemes, the harvested power is less than our proposed robust scheme since the transmitter cannot utilize the available degrees of freedom when we set the energy beams and AN in the null space of channel $\mathbf{h}$ and fix $\mathbf{W}$ for MRT.

\subsection{Average total transmit power}

Figure 6 depicted the average total transmit power versus minimum required SINR of IR. To compare clearly, we set the non-robust scheme as the benchmark scheme. In particular, with regard to the non-robust scheme, we calculate the average minimum harvested power at per ER under different minimum required SINR of IR with fixed

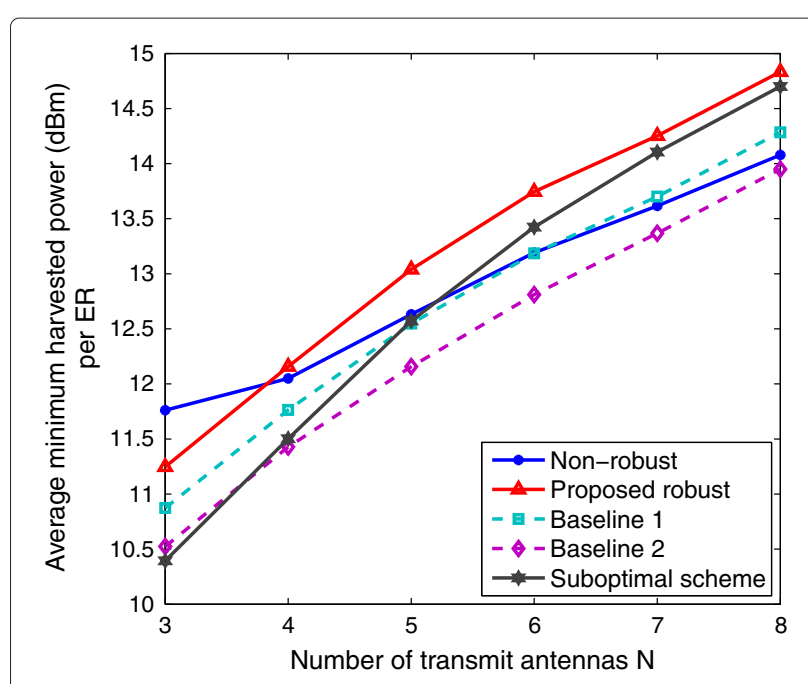

Fig. 5 Simulation 4. Average minimum harvested power per ER versus number of transmit antennas $\mathrm{N}$ 


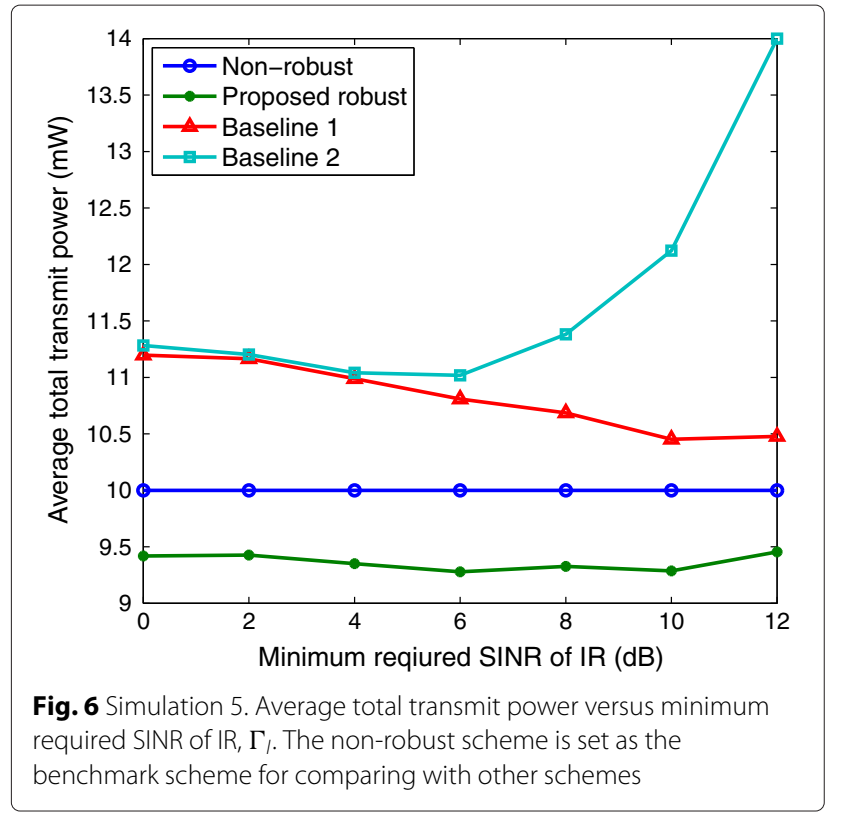

maximum total transmit power $P=10 \mathrm{~mW}$. Then, by setting the result value of harvested power as the benchmark level of three other schemes under their relative minimum required SINR of IR, we obtain the average total transmit power of the three other schemes. It is obvious that the benchmark level of harvested power decreases with increasing minimum required SINR of IR. As seen from this figure, the consumed transmit power of our proposed robust scheme is apparently lower than the non-robust scheme. Moreover, it changes slightly owning to the tradeoff between required SINR of IR and power harvesting at per ER. On the contrary, the two baseline schemes consume higher transmit power than our proposed robust scheme since the transmitter is unable to fully make use of the degrees of freedom for both the energy beam matrix $\mathbf{V}$ and AN covariance matrix $\mathbf{V}_{0}$ which lie in the null space of channel h. Meanwhile, $\mathbf{W}$ is fixed. By optimizing the power splitting ratio $\rho$, baseline scheme 1 saves much transmit power compare with baseline scheme 2 with increasing required SINR of IR.

\subsection{Secrecy capacity}

Figure 7 shows the average secrecy capacity of IR versus minimum required SINR of IR. It is expected that the secrecy capacity of IR increases with minimum required SINR of IR since the secrecy capacity is a monotonous increasing function w.r.t. SINR. On the other hand, our proposed robust scheme ensures no worse secrecy capacity than the non-robust scheme by the time that the former provides substantial performance gain in terms of power harvesting compared to the latter. It can be seen that both the proposed robust and non-robust schemes achieve nearly the same secrecy capacity for they satisfy the boundary condition (constraints $\mathrm{C} 1$ and $\mathrm{C} 2$ ) of the

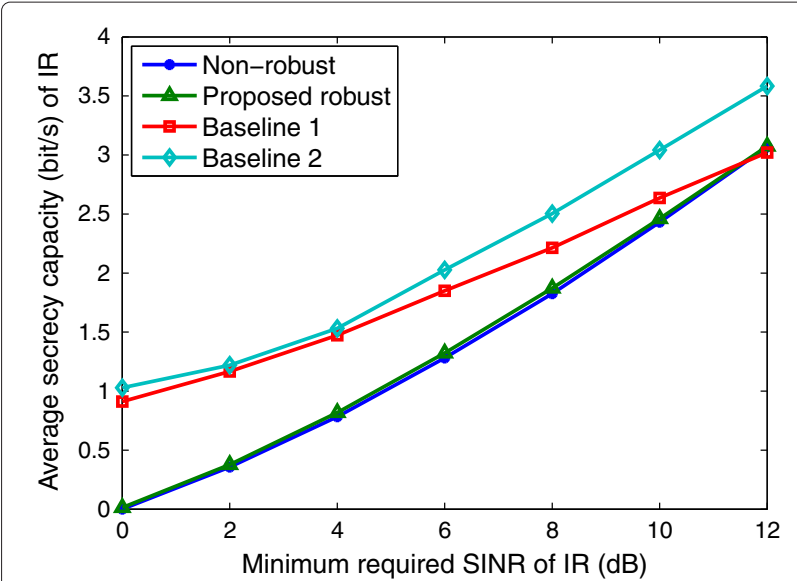

Fig. 7 Simulation 6. Average secrecy capacity versus minimum required SINR of IR, $\Gamma$,

QoS requirement on communication. Besides, the two baseline schemes obtain average higher secrecy capacity compared to the other schemes. Nevertheless, from Fig. 6, we learn that the excellent performance in secrecy capacity stems from the consuming of a very high total transmit power.

\section{Conclusions}

An optimal robust secrecy beamforming design for MISO communication system with SWIPT was investigated in this paper. In order to solve the formulated original optimization problem efficiently, we converted it into a convex SDR problem and proved the tightness of this adopted SDR. As a result, the obtained power splitting ratio and the transmit beamforming matrices based on the worstcase max-min fair energy harvesting among $K$ ERs are the global optimal solution. In addition, a suboptimal scheme was proposed with lower computational complexity. Simulation results demonstrated the superior performance of our proposed schemes compared to the other schemes.

\section{Appendix 1 \\ Proof of Theorem 1}

The main idea of this proof lies in KKT condition [17] and rank analysis of matrices. The Lagrange dual function of (7) is modeled as

$$
\begin{aligned}
\mathcal{L}(\mathbf{X})= & t+\sum_{k=1}^{K} \operatorname{Tr}\left(\mathbf{X}_{k} \mathbf{M}_{\mathrm{ER}, k}\left(\mathbf{W}, \mathbf{V}, \mathbf{V}_{0}, \lambda_{k}\right)\right) \\
& +\sum_{k=1}^{K} \operatorname{Tr}\left(\mathbf{Y}_{k} \mathbf{T}_{\mathrm{ER}, k}\left(\mathbf{W}, \mathbf{V}_{0}, \tilde{\lambda}_{k}\right)\right) \\
& +\alpha\left[P-\operatorname{Tr}\left(\mathbf{W}+\mathbf{V}+\mathbf{V}_{0}\right)\right] \\
& +\beta\left[\operatorname{Tr}\left(\left(\mathbf{W}-r \mathbf{V}-r \mathbf{V}_{0}\right) \mathbf{H}\right)-\frac{r \sigma_{I}^{2}}{\rho}\right] \\
& +\gamma\left[\operatorname{Tr}\left(\left(\mathbf{W}+\mathbf{V}+\mathbf{V}_{0}\right) \mathbf{H}\right)-\frac{P_{\min }}{(1-\rho) \eta}\right] \\
& +\operatorname{Tr}(\Phi \mathbf{W})+\operatorname{Tr}(\Xi \mathbf{V})+\operatorname{Tr}\left(\Omega \mathbf{V}_{0}\right),
\end{aligned}
$$


where $\mathbf{X}=\left\{\mathbf{W}, \mathbf{V}, \mathbf{V}_{0}, \lambda_{k}, \tilde{\lambda}_{k}, \mathbf{X}_{k}, \mathbf{Y}_{k}, \alpha, \beta, \gamma, \Phi, \Xi, \Omega\right\}$ includes all the primal and dual variables, and $\mathbf{X}_{k} \in$ $\mathbb{H}_{+}^{N M+1}, \forall k, \mathbf{Y}_{k} \in \mathbb{H}_{+}^{N M+1}, \forall k, \alpha \in \mathbb{R}_{+}, \beta \in \mathbb{R}_{+}$ and $\gamma \in \mathbb{R}_{+}$are the dual variables with respect to $\mathbf{M}_{\mathrm{ER}, k}\left(\mathbf{W}, \mathbf{V}, \mathbf{V}_{0}, \lambda_{k}\right), \mathbf{T}_{\mathrm{ER}, k}\left(\mathbf{W}, \mathbf{V}_{0}, \tilde{\lambda}_{k}\right), \mathrm{C} 4, \mathrm{C} 1$, and C3, respectively. $\Phi \in \mathbb{H}_{+}^{N}, \Xi \in \mathbb{H}_{+}^{N}$, and $\Omega \in \mathbb{H}_{+}^{N}$ are the dual variables regard to $\mathbf{W}, \mathbf{V}$, and $\mathbf{V}_{0}$, respectively.

For the convenience of expression, we re-express $\mathbf{M}_{\mathrm{ER}, k}\left(\mathbf{W}, \mathbf{V}, \mathbf{V}_{0}, \lambda_{k}\right)$ and $\mathbf{T}_{\mathrm{ER}, k}\left(\mathbf{W}, \mathbf{V}_{0}, \tilde{\lambda}_{k}\right)$ as

$$
\begin{aligned}
& \mathbf{M}_{\mathrm{ER}, k}\left(\mathbf{W}, \mathbf{V}, \mathbf{V}_{0}, \lambda_{k}\right)=\tilde{\mathbf{G}}_{k}^{H}\left(\overline{\mathbf{W}}+\overline{\mathbf{V}}+\overline{\mathbf{V}}_{0}\right) \tilde{\mathbf{G}}_{k}+\Lambda_{e, k}, \\
& \mathbf{T}_{\mathrm{ER}, k}\left(\mathbf{W}, \mathbf{V}_{0}, \tilde{\lambda}_{k}\right)=\tilde{\mathbf{G}}_{k}^{H}\left(r_{k} \overline{\mathbf{V}}_{0}-\overline{\mathbf{W}}\right) \tilde{\mathbf{G}}_{k}+\tilde{\Lambda}_{e, k},
\end{aligned}
$$

where

$$
\begin{aligned}
\tilde{\mathbf{G}}_{k}= & {\left[\begin{array}{ll}
\mathbf{I}_{N M} & \overline{\mathbf{g}}_{k}
\end{array}\right] } \\
\Lambda_{e, k} & =\left[\begin{array}{cc}
\lambda_{k} \mathbf{I}_{N M} & \mathbf{0} \\
\mathbf{0} & -\frac{t}{\eta}-\lambda_{k} \varepsilon_{e, k}^{2}
\end{array}\right], \\
\tilde{\Lambda}_{e, k} & =\left[\begin{array}{cc}
\tilde{\lambda}_{k} \mathbf{I}_{N M} & \mathbf{0} \\
\mathbf{0} & r_{k} \sigma_{E}^{2}-\tilde{\lambda}_{k} \varepsilon_{e, k}^{2}
\end{array}\right] .
\end{aligned}
$$

To facilitate the analysis of the construction of the resulting solution, $\overline{\mathbf{W}}, \overline{\mathbf{V}}$, and $\overline{\mathbf{V}}_{0}$ must be written back into their primal styles. Therefore, we specify

$$
\begin{gathered}
\tilde{\mathbf{G}}_{k} \mathbf{X}_{k} \tilde{\mathbf{G}}_{k}^{H}=\left[\begin{array}{ccc}
\mathbf{Z}_{e, k}^{(1,1)} & \cdots & \mathbf{Z}_{e, k}^{(1, M)} \\
\vdots & \ddots & \vdots \\
\mathbf{Z}_{e, k}^{(M, 1)} & \cdots & \mathbf{Z}_{e, k}^{(M, M)}
\end{array}\right] \in \mathbb{H}_{+}^{N M}, \\
\tilde{\mathbf{G}}_{k} \mathbf{Y}_{k} \tilde{\mathbf{G}}_{k}^{H}=\left[\begin{array}{ccc}
\mathbf{U}_{e, k}^{(1,1)} & \cdots & \mathbf{U}_{e, k}^{(1, M)} \\
\vdots & \ddots & \vdots \\
\mathbf{U}_{e, k}^{(M, 1)} & \cdots & \mathbf{U}_{e, k}^{(M, M)}
\end{array}\right]_{\mathbf{U}^{(l, l)}}^{(l, l)}, \in \mathbb{H}_{+}^{N}, \\
\end{gathered}
$$

Substituting (15) and (16) into (14), the Lagrange dual function could be reformulated as

$$
\begin{aligned}
\mathcal{L}(\mathbf{X})= & t+\sum_{k=1}^{K} \operatorname{Tr}\left[\sum_{l=1}^{M}\left(\mathbf{W}+\mathbf{V}+\mathbf{V}_{0}\right) \mathbf{Z}_{e, k}^{(l, l)}+\mathbf{X}_{k} \Lambda_{E, K}\right] \\
& +\sum_{k=1}^{K} \operatorname{Tr}\left[\sum_{l=1}^{M}\left(r_{k} \mathbf{V}_{0}-\mathbf{W}\right) \mathbf{U}_{e, k}^{(l, l)}+\mathbf{Y}_{k} \tilde{\Lambda}_{E, K}\right] \\
& +\alpha\left[P-\operatorname{Tr}\left(\mathbf{W}+\mathbf{V}+\mathbf{V}_{0}\right)\right] \\
& +\beta\left[\operatorname{Tr}\left(\left(\mathbf{W}-r \mathbf{V}-r \mathbf{V}_{0}\right) \mathbf{H}\right)-\frac{r \sigma_{I}^{2}}{\rho}\right] \\
& +\gamma\left[\operatorname{Tr}\left(\left(\mathbf{W}+\mathbf{V}+\mathbf{V}_{0}\right) \mathbf{H}\right)-\frac{P_{\min }}{(1-\rho) \eta}\right] \\
& +\operatorname{Tr}[\Phi \mathbf{W}]+\operatorname{Tr}[\mathbf{\Xi} \mathbf{V}]+\operatorname{Tr}\left[\mathbf{U V}_{0}\right] .
\end{aligned}
$$

It is verified that the SDR problem (7) is jointly convex with respect to the primal variables and satisfies the Slater's condition [17]. Thus, the gap between the primal and dual optimization problem is zero. According to (17), the dual optimization problem is given by

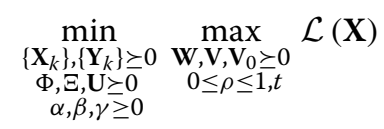

which achieves the same objective value with its relative primal optimization problem (7). Through it, we obtain $\left\{\mathbf{W}^{*}, \mathbf{V}^{*}, \mathbf{V}_{0}^{*}, \rho^{*}, t^{*}\right\}$ and $\left\{\mathbf{X}_{k}^{*}, \mathbf{Y}_{k}^{*}, \Phi^{*}, \mathbf{\Xi}^{*}, \mathbf{U}^{*}, \alpha^{*}, \beta^{*}, \gamma^{*}\right\}$ as the primal and dual optimal solution of (7) and (18), respectively. Base on the Karush-Kuhn-Tucker (KKT) conditions [17], the equations $\mathbf{A}_{1}^{*}+\Phi^{*}=$ $\mathbf{0}, \mathbf{A}_{1}^{*} \mathbf{W}^{*}=\mathbf{0}$ and $\Phi^{*} \mathbf{W}^{*}=\mathbf{0}$ hold, where $\mathbf{A}_{1}^{*}=\sum_{k=1}^{K} \sum_{l=1}^{M}\left(\mathbf{Z}_{e, k}^{*(l, l)}-\mathbf{U}_{e, k}^{(* l, l)}\right)+\left(\beta^{*}+\gamma^{*}\right) \mathbf{H}-\alpha^{*} \mathbf{I}_{N}$. Meanwhile, we obtain $\rho^{*}=\frac{\sqrt{\beta^{*} \eta r \sigma_{I}^{2}}}{\sqrt{\gamma^{*} P_{\min }}+\sqrt{\beta^{*} \eta r \sigma_{I}^{2}}}$. For the case that $\Phi^{*} \succeq \mathbf{0}$, so we have $\mathbf{A}_{1}^{*} \preceq \mathbf{0}$. Define

$$
\mathbf{B}_{1}^{*}=-\alpha^{*} \mathbf{I}_{N}+\sum_{k=1}^{K} \sum_{l=1}^{M}\left(\mathbf{z}_{e, k}^{*(l, l)}-\mathbf{U}_{e, k}^{*(l, l)}\right),
$$

then we obtain

$$
\mathbf{A}_{1}^{*}=\mathbf{B}_{1}^{*}+\left(\beta^{*}+\gamma^{*}\right) \mathbf{H} .
$$

Without loss of generality, we define $r_{1}=\operatorname{rank}\left(\mathbf{B}_{1}^{*}\right)$. Then, two cases are considered of $r_{1}$ to help our analysis of $\mathbf{A}_{1}^{*}$. Firstly, we assume that $r_{1}=N$, i.e., the matrix $\mathbf{B}_{1}^{*}$ is of full rank. Upon that, we have $\operatorname{rank}\left(\mathbf{A}_{1}^{*}\right)=\operatorname{rank}\left(\mathbf{B}_{1}^{*}+\left(\beta^{*}+\gamma^{*}\right) \mathbf{H}\right) \geq \operatorname{rank}\left(\mathbf{B}_{1}^{*}\right)-$ $\operatorname{rank}\left(\left(\beta^{*}+\gamma^{*}\right) \mathbf{H}\right)=N-1$. However, $\operatorname{rank}\left(\mathbf{A}_{1}^{*}\right)=N$ is undesirable because according to $\mathbf{A}_{1}^{*} \mathbf{W}^{*}=\mathbf{0}$, it follows that $\mathbf{W}^{*}=\mathbf{0}$ which, of course, is not the optimal solution to (7). Such that, we choose $\operatorname{rank}\left(\mathbf{A}_{1}^{*}\right)=N-1$ and define $\tau_{1} \in \mathbb{C}^{N \times 1}$, with unit norm, spans the null space of $\mathbf{A}_{1}^{*}$. Then, we get the optimal solution $\mathbf{W}^{*}=f \tau_{1} \tau_{1}{ }^{H}, f \geq 0$. Secondly, we consider $r_{1}<N$, i.e., the matrix $\mathbf{B}_{1}^{*}$ is rank defective. In this case, we model $\mathbf{N}_{1} \in \mathbb{C}^{N \times\left(N-r_{1}\right)}$ as the orthogonal basis of the null space of $\mathbf{B}_{1}^{*}$, i.e., null $\left(\mathbf{B}_{1}^{*}\right)=$ $\mathbf{N}_{1}, \mathbf{B}_{1}^{*} \mathbf{N}_{1}=\mathbf{0}$, and $\operatorname{rank}\left(\mathbf{N}_{1}\right)=N-r_{1}$. Also, we set $\pi_{1, n} \in \mathbb{C}^{N \times 1}, 1 \leq n \leq N-r_{1}$, as the $n$th column of matrix $\mathbf{N}_{1}$. Then, we obtain the equality

$$
\begin{aligned}
\pi_{1, n}^{H} \mathbf{A}_{1}^{*} \pi_{1, n} & =\pi_{1, n}^{H}\left(\mathbf{B}_{1}^{*}+\left(\beta^{*}+\gamma^{*}\right) \mathbf{H}\right) \pi_{1, n} \\
& =\left(\beta^{*}+\gamma^{*}\right) \pi_{1, n}^{H} \mathbf{H} \pi_{1, n} \\
& =\left(\beta^{*}+\gamma^{*}\right) \pi_{1, n}^{H} \mathbf{h} \mathbf{h}^{H} \pi_{1, n} .
\end{aligned}
$$

Owing to $\mathbf{A}_{1}^{*} \preceq \mathbf{0}$ and $\left|\pi_{1, h}^{H} \mathbf{h}\right| \geq 0$, it results that

$$
\mathbf{A}_{1}^{*} \mathbf{N}_{1}=\mathbf{0} \text { and } \mathbf{H} \mathbf{N}_{1}=\mathbf{0},
$$


i.e., $\mathbf{N}_{1}$ lies in the null space of both $\mathbf{A}_{1}^{*}$ and $\mathbf{H}$. Since $\operatorname{rank}\left(\mathbf{N}_{1}\right)=N-r_{1}$, it deduces that $\operatorname{rank}\left(\mathbf{A}_{1}^{*}\right) \leq N-$ $\left(N-r_{1}\right)=r_{1}$. What is more, according to (20), we achieve another inequality: $\operatorname{rank}\left(\mathrm{A}_{1}^{*}\right) \geq \operatorname{rank}\left(\mathbf{B}_{1}^{*}\right)-$ $\operatorname{rank}(\mathbf{H})=r_{1}-1$. Finally, $\operatorname{rank}\left(\mathbf{A}_{1}^{*}\right)$ is bounded by $r_{1}-1 \leq$ $\operatorname{rank}\left(\mathbf{A}_{1}^{*}\right) \leq r_{1}$. Following with this, the rank of $\mathbf{W}^{*}$ can be bounded between $N-r_{1} \leq \operatorname{rank}\left(\mathbf{W}^{*}\right) \leq N-r_{1}+1$.

Now, we come to discuss about rank $\left(\mathbf{W}^{*}\right)$, i.e., whether it equals to $N-r_{1}$ or $N-r_{1}+1$. Suppose that rank $\left(\mathbf{W}^{*}\right)=$ $N-r_{1}$, which equals to $\operatorname{rank}\left(\mathbf{N}_{1}\right)$; then, we have $\mathbf{W}^{*}=$ $\mathbf{N}_{1}$ and express $\mathbf{W}^{*}$ as $\mathbf{W}^{*}=\sum_{n=1}^{N-r_{1}} \mu_{n} \pi_{1, n} \pi_{1, n}^{H}$, where $\mu_{n} \geq 0, \forall n$. Nevertheless, due to (22), $\operatorname{Tr}\left(\mathbf{H W}^{*}\right)=$ $N-r_{1}$

$\sum_{n=1}^{N-r_{1}} \mu_{n} \operatorname{Tr}\left(\mathbf{H} \pi_{1, n} \pi_{1, n}^{H}\right)=0$ holds which violates the SINR of IR $\Gamma_{I}>0$. Thus, it remains $\operatorname{rank}\left(\mathbf{W}^{*}\right)=N-r_{1}+1$. According to what we have defined the unique unit norm vector $\tau_{1} \in \mathbb{C}^{N \times 1}$ which lie in the null space of $\mathbf{A}_{1}^{*}$, it also satisfies $\mathbf{N}_{1} \tau_{1}=\mathbf{0}$. So, we obtain

$$
\mathbf{W}^{*}=\left[\begin{array}{ll}
\mathbf{N}_{1} & \tau_{1}
\end{array}\right] \text {, }
$$

and the optimal solution of (7) can be modeled as

$$
\mathbf{W}^{*}=\sum_{n=1}^{N-r_{1}} \mu_{n} \pi_{1, n} \pi_{1, n}^{H}+f \tau_{1} \tau_{1}^{H},
$$

where $\mu_{n} \geq 0, \forall n$ and $f>0$. The first part of Theorem 1 is thus proved.

Next, we proof the second part of Theorem 1. Suppose we achieved the optimal solution $\left\{\mathbf{W}^{*}, \mathbf{V}^{*}, \mathbf{V}_{0}^{*}, \rho^{*}, t^{*}\right\}$ of (7), where the $\mathbf{W}^{*}$ is given in (9) and $\mu_{n}>0$, i.e., $\operatorname{rank}\left(\mathbf{W}^{*}\right)>1$. Then, an alternative solution $\left\{\widetilde{\mathbf{W}}^{*}, \widetilde{\mathbf{V}}^{*}, \widetilde{\mathbf{V}}_{0}^{*}, \tilde{\rho}^{*}, \tilde{t}^{*}\right\}$ can be constructed as given in (10)(12) and it has the following properties:

$$
\begin{aligned}
& \operatorname{Tr}\left[\left(\widetilde{\mathbf{W}}^{*}-r \widetilde{\mathbf{V}}^{*}-r \widetilde{\mathbf{V}}_{0}^{*}\right) \mathbf{H}\right] \\
& =\operatorname{Tr}\left[\left(\mathbf{W}^{*}-r \mathbf{V}^{*}-r \mathbf{V}_{0}^{*}-(r+1) \sum_{n=1}^{N-r_{1}} \mu_{n} \pi_{1, n} \pi_{1, n}^{H}\right) \mathbf{H}\right] \\
& =\operatorname{Tr}\left[\left(\mathbf{W}^{*}-r \mathbf{V}^{*}-r \mathbf{V}_{0}^{*}\right) \mathbf{H}\right] \geq \frac{r \sigma_{I}^{2}}{\tilde{\rho}^{*}}
\end{aligned}
$$$$
\operatorname{Tr}\left[\mathbf{G}_{k}^{H}\left(\widetilde{\mathbf{W}}^{*}-r_{k} \widetilde{\mathbf{V}}_{0}^{*}\right) \mathbf{G}_{k}\right]
$$$$
=\operatorname{Tr}\left[\mathbf{G}_{k}^{H}\left(\mathbf{W}^{*}-\sum_{n=1}^{N-r_{1}} \mu_{n} \pi_{1, n} \pi_{1, n}^{H}-r_{k} \mathbf{V}_{0}^{*}\right) \mathbf{G}_{k}\right]
$$$$
\leq \operatorname{Tr}\left[\mathbf{G}_{k}^{H}\left(\mathbf{W}^{*}-r_{k} \mathbf{V}_{0}^{*}\right) \mathbf{G}_{k}\right] \leq r_{k} \sigma_{k}^{2}, \forall k \text {, }
$$

$$
\begin{aligned}
& \operatorname{Tr}\left[\left(\widetilde{\mathbf{W}}^{*}+\tilde{\mathbf{V}}^{*}+\widetilde{\mathbf{V}}_{0}^{*}\right) \mathbf{H}\right] \\
& \quad=\operatorname{Tr}\left[\left(\mathbf{W}^{*}+\mathbf{V}^{*}+\mathbf{V}_{0}^{*}\right) \mathbf{H}\right] \geq \frac{P_{\min }}{\left(1-\tilde{\rho}^{*}\right) \eta} \\
& \operatorname{Tr}\left(\tilde{\mathbf{W}}^{*}+\tilde{\mathbf{V}}^{*}+\tilde{\mathbf{V}}_{0}^{*}\right)=\operatorname{Tr}\left(\mathbf{W}^{*}+\mathbf{V}^{*}+\mathbf{V}_{0}^{*}\right) \leq P
\end{aligned}
$$

\section{Competing interests}

The authors declare that they have no competing interests.

\section{Acknowledgements}

This paper was supported by the National Natural Science Foundation of China (No. 61271232, 61372126); the Open research fund of National Mobile Communications Research Laboratory, Southeast University (No. 2012D05); the Priority Academic Program Development of Jiangsu Province (Smart Grid and Control Technology).

\section{Author details}

${ }^{1}$ College of Communication and Information, Nanjing University of Posts and Telecommunications, Xinmofan Road 66, 210003 Nanjing, China. ${ }^{2}$ National Mobile Communications Research Laboratory, Southeast University, Nanjing, China.

Received: 16 December 2014 Accepted: 10 May 2015

Published online: 09 June 2015

The properties from (25) to (30) demonstrate that the

\section{Appendix 2}

Proof of Proposition 1

where $\Phi^{*} \mathbf{W}^{*}=\mathbf{0}$ owing to the complementary slackness condition. We perform a basic rank operation with regard to (32) which obtains

$$
=\min \left\{\operatorname{rank}\left(\left(\beta^{*}+\gamma^{*}\right) \mathbf{H}\right), \operatorname{rank}\left(\mathbf{W}^{*}\right)\right\} .
$$




\section{References}

1. R Zhang, $\mathrm{CHo}, \mathrm{MIMO}$ broadcasting for simultaneous wireless information and power transfer. IEEE Trans. Wireless Commun. 12(5), 1989-2001 (2013)

2. $X$ Zhou, R Zhang, CK Ho, Wireless information and power transfer: architecture design and rate-energy tradeoff. 11. 61, 4754-4767 (2013)

3. L Liu, R Zhang, C Ho, Secrecy wireless information and power transfer with MISO beamforming. IEEE Trans. Signal Process. 62(7), 1850-1863 (2014)

4. L Liu, R Zhang, KC Chua, Wireless information transfer with opportunistic energy harvesting. IEEE Trans. Wireless Commun. 12(1), 288-300 (2013)

5. Q Shi, W Xu, L Liu, R Zhang, Joint transmit beamforming and receive power splitting for MISO SWIPT systems. IEEE Trans. Wireless Commun. 13(6), 3269-3280 (2014)

6. DWK Ng, Schober Lo E S R, Robust beamforming for secure communication in systems with wireless information and power transfer. IEEE Trans. Wireless Commun. 13(8), 4599-4615 (2014)

7. R Feng, Q Li, Q Zhang, Robust secure transmission in MISO simultaneous wireless information and power transfer system. IEEE Trans. Vehicular Technol. 64(1), 400-405 (2014)

8. MRA Khandaker, K-K Wong, Robust secrecy beamforming with energy-harvesting eavesdroppers. IEEE Wireless Commun. Lett. 4(1), 10-13 (2014)

9. DWK Ng, R Schober, Max-min fair wireless energy transfer for secure multiuser communication systems. IEEE Information Theory Workshop (ITW), 326-330 (2014)

10. J Huang, AL Swindlehurst, Robust secure transmission in MISO channels based on worst-case optimization. IEEE Trans. Signal Process. 60(4), 1696-1707 (2012)

11. K Huang, E Larsson, Simultaneous information and power transfer for broadband wireless systems. IEEE Trans. Signal Process. 23, 5972-5986 (2013)

12. Y Huang, DP Palomar, Rank-constrained separable semidefinite program with applications to optimal beamforming. IEEE Trans. Signal Process. 58(2), 664-678 (2010)

13. $\mathrm{Q} \mathrm{Li}, \mathrm{W}-\mathrm{K}$ Ma, Spatially selective artificial-noise aided transmit optimization for MISO multi-eves secrecy rate maximization. IEEE Trans. Signal Process. 61(10), 2704-2717 (2013)

14. J Xu, L Liu, R Zhang, in IEEE International Conference on Acoustics, Speech, and Signal Processing (ICASSP). Multiuser MISO beamforming for simultaneous wireless information and power transfer, (2013), pp. 4754-4758

15. Z Luo, W-K Ma, M-C ASo, Y Ye, S Zhang, Semidefinite relaxation of quadratic optimization problems. IEEE Signal Process. Mag. 27(3), 20-34 (2010)

16. Z-Q Luo, S Zhang, Dynamic spectrum management: complexity and duality. IEEE J. Sel. Topics Signal Process. 2(1), 57-73 (2008)

17. S Boyd, L Vandenberghe, Convex Optimization. (Cambridge University Press, 2009)

18. KC Toh, MJ Todd, RH Tutuncu, SDPT3 - A Matlab software package for semidefinite programming, version 1.3. Optimization Methods and Softw. 11(1-4), 545-581 (1999)

19. JF Sturm, Using SeDuMi 1.02, A MATLAB toolbox for optimization over symmetric cones. Optimiz. Methods and Softw. 11(1-4), 625-653 (1999)

20. S Goel, R Negi, Guaranteeing secrecy using artificial noise. IEEE Trans. Wireless Commun. 7(6), 2180-2189 (2008)

\section{Submit your manuscript to a SpringerOpen ${ }^{\circ}$ journal and benefit from:}

- Convenient online submission

- Rigorous peer review

- Immediate publication on acceptance

- Open access: articles freely available online

- High visibility within the field

- Retaining the copyright to your article

Submit your next manuscript at $>$ springeropen.com 